\title{
MINERALIZAÇÃO DO RESÍDUO DA PUPUNHEIRA EM CONDIÇÕES DE
} CAMPO E LABORATÓRIO

Doi:http://dx.doi.org/10.1590/1809-4430-Eng.Agric.v35n5p918-930/2015

\section{MAGNO S. PEREIRA ${ }^{1}$, ANTONIO T. MATOS ${ }^{2}$, ALISSON C. BORGES ${ }^{3}$, MICHAEL F. NUNES ${ }^{4}$}

RESUMO: Objetivou-se, neste trabalho, estudar o processo de decomposição do carbono (CO) e nitrogênio orgânico (NO) dos resíduos da pupunheira, em condições de campo e laboratório, e em duas formas de aplicação: com incorporação ou em disposição superficial no solo. $\mathrm{O}$ experimento de campo foi conduzido em Cambissolo Háplico Tb distrófico latossólico (CXbd), nas condições climáticas tropicais do período de primavera/verão, na cidade de Viçosa-MG, Brasil. Os resíduos foram incubados em condição de campo e laboratório, durante 112 dias, período no qual foram retiradas amostras para a análise das concentrações mineralizadas do $\mathrm{CO}$ e NO. A degradação do resíduo foi mais intensa em condição de campo que na de laboratório, e a incorporação do resíduo no solo acelerou a degradação nas duas condições de incubação do material. Os métodos utilizados para a estimativa dos coeficientes e frações de mineralização do $\mathrm{CO}$ e disponibilização do nitrogênio inorgânico, em condição de laboratório, geraram resultados condizentes com os obtidos na condição de campo, contudo subestimaram consideravelmente os valores obtidos.

PALAVRAS-CHAVE: degradação, matéria orgânica, fração de decomposição.

\section{MINERALIZATION OF PEACH PALM WASTE UNDER FIELD AND LABORATORY CONDITIONS}

\begin{abstract}
The objective of this study was to evaluate organic carbon (OC) and organic nitrogen (ON) decomposition in peach palm waste under field and laboratory conditions. Application was performed by both incorporating and releasing the waste onto soil surface. Field experiment was carried out on an Inceptisol under tropical conditions during the spring and summer seasons in Viçosa - MG, Brazil. The waste was incubated under field and laboratory conditions for 112 days within which samples were taken for analysis. We noted that waste was more intensely degraded under field conditions than in lab environment. Moreover, waste incorporation into soil accelerated the degradation under both incubation conditions. The estimation methods for coefficients and fractions of OC mineralization, inorganic nitrogen availability under lab condition produced results consistent with field ones; however, they were considerably underestimated.
\end{abstract}

KEYWORDS: Degradation, Organic matter, Fraction of decomposition.

\section{INTRODUÇÃO}

O Brasil é o maior produtor e consumidor de palmito do mundo, e o cultivo da pupunheira para produção de palmito vem despertando, desde a década de 1970, o interesse de agricultores de todo o País, devido principalmente à demanda elevada, tanto interna quanto externa, de palmito de boa qualidade, e à alta lucratividade do setor (SOUSA et al., 2011). No entanto, para a extração e beneficiamento do palmito da pupunheira nas agroindústrias, grandes quantidades de resíduos são

\footnotetext{
${ }_{1}^{1}$ Doutorando em Engenharia Agrícola, Departamento de Engenharia Agrícola, UFV/Viçosa - MG, Fone: (31) 3899-1868, magno.eaa@gmail.com

${ }^{2}$ Eng ${ }^{\circ}$ Agrícola, Prof. Doutor, Departamento de Engenharia Sanitária e Ambiental, UFMG/ Belo Horizonte - MG, atmatos@desa.ufmg.br

${ }^{3}$ Eng $^{\circ}$ Civil, Prof. Doutor, Departamento de Engenharia Agrícola, UFV/Viçosa - MG, borges@ufv.br

${ }^{4}$ Graduando em Engenharia Agrícola e Ambiental, Departamento de Engenharia Agrícola, UFV/Viçosa -

MG, michael.nunes@ufv.br 
gerados (70\% do material original), com uma produção mensal, por indústria, estimada em torno de 135 toneladas de resíduo por mês, constantemente ao longo do ano, totalizando 12.576 toneladas por ano somente na região do Vale do Ribeira - SP (MORAES et al., 2011).

O interesse no uso de resíduos orgânicos na agricultura brasileira está fundamentado nos elevados teores de carbono em compostos orgânicos e de nutrientes, além do aumento na CTC e da neutralização da acidez que estes podem proporcionar ao solo (MATOS, 2014). Segundo MATOS (2010), o uso de resíduos orgânicos na agricultura como fertilizante, além de disponibilizar macro e micronutrientes, também representa proteção ambiental e conservação de recursos naturais, uma vez que, dentre outros benefícios, a correta disposição dos resíduos no solo proporciona melhoria da estruturação e da estabilização dos agregados, aumento da porosidade total, melhoria das condições físico-hídricas, aumento da capacidade de retenção de água e complexação/quelatação de substâncias tóxicas.

A disposição e o tratamento de resíduos devem ser considerados fases a serem incluídas no processo produtivo, e a busca por procedimentos de descarte corretos pode levar à redução nos impactos ambientais e a prejuízos resultantes de multas e/ou má gestão dos recursos naturais (SILVA et al., 2012). A prática da disposição no solo exige, no entanto, constante monitoramento da disponibilização e do acúmulo de nutrientes no solo, já que a aplicação de quantidades acima da capacidade de suporte do solo ou da necessidade das culturas pode gerar problemas ambientais associados às perdas de nitrogênio, aumento na população de patógenos e vetores, e na concentração de metais pesados do solo ou salinizar solos agrícolas. Também é importante pesquisar se a fertilização com tais resíduos pode ser comparável à adubação mineral em termos de produtividade e de qualidade das culturas (TASSO J. et al., 2007).

Diversos fatores interferem na decomposição e na mineralização dos resíduos orgânicos, dentre os quais se podem citar a relação $\mathrm{C} / \mathrm{N}$ dos resíduos, as características físico-químicas e biológicas, além da temperatura e da umidade do solo (FIGUEIREDO et al., 2012). Os coeficientes de degradação do carbono e do nitrogênio orgânicos podem ser obtidos em condições de laboratório. Nesse caso, tem sido comum a utilização de câmaras (respirômetros), equipamentos que possibilitam que amostras da mistura de solo mais resíduo e somente solo (controle) sejam incubadas por períodos variáveis, sendo assim medidas periodicamente as quantidades mineralizadas de carbono emanada na forma de $\mathrm{CO}_{2}$ ou de nitrogênio nas formas de $\mathrm{NH}_{4}{ }^{+} \mathrm{e} \mathrm{NO}_{3}{ }^{-}$ (BARROS et al., 2010; FIORESE et al., 2012; MARIANO et al., 2013).

A degradação do material orgânico e a disponibilização de nitrogênio mineralizado, em função do tempo, podem ser estimadas com a utilização de modelos matemáticos (BOEIRA et al., 2011). Comumente, a dinâmica na mineralização da matéria orgânica de resíduos aplicados em solos tem sido expressa por uma equação de cinética de reação de primeira ordem (STANFORD \& SMITH, 1972).

Em decorrência da falta de informações e da disponibilidade de modelos matemáticos que possibilitem a estimativa da disponibilização de nutrientes, realizou-se este trabalho com o objetivo de monitorar a dinâmica do carbono e do nitrogênio orgânico durante o processo de mineralização do resíduo orgânico da pupunheira, em condições de campo e laboratório, quando incorporado ou disposto sobre o solo, além de ajustar modelos matemáticos de cinética de primeira ordem para a representação dos processos.

\section{MATERIAL E MÉTODOS}

Os experimentos foram conduzidos em condição de campo, em Cambissolo Háplico Tb distrófico latossólico (CXbd), na Área Experimental de Tratamento de Resíduos Urbanos (latitude $20^{\circ} 46^{\prime} 20^{\prime}$ S, longitude $42^{\circ} 52^{\prime} 19^{\prime}$ ' W, altitude de $677 \mathrm{~m}$ ) e em condição de laboratório, no Laboratório de Solos e Resíduos Sólidos do Departamento de Engenharia Agrícola da Universidade Federal de Viçosa - DEA/UFV. Os resíduos da pupunheira foram obtidos no Sítio Barra Alegre, 
localizado no município de Descoberto-MG, onde há o cultivo da pupunheira com a finalidade de comercialização do palmito na forma minimamente processada ou in natura.

Os resíduos (bainha, base e ponta) foram coletados e passados em picadeira comercial de forragem, gerando-se um material com granulometria de 3 a $6 \mathrm{~mm}$ e, posteriormente, misturados manualmente na proporção estimada em que foram gerados, compondo-se um resíduo misto das três partes. Do resíduo misto foram retiradas amostras para sua caracterização física e química, seguindo-se o método descrito por MATOS (2012), cujos resultados estão expostos na Tabela 1.

TABELA 1. Características químicas e físicas do resíduo misto fresco e seco. Chemical and physical characteristics of fresh and dry mixed waste.

\begin{tabular}{|c|c|c|c|}
\hline Variáveis $^{(1)}$ & Unidade & Matéria Fresca & Matéria Seca \\
\hline $\mathrm{CO}_{\mathrm{fo}}$ & dag $\mathrm{kg}^{-1}$ & 6,01 & 41,85 \\
\hline $\operatorname{COT}_{(\text {v.u. })}$ & dag $\mathrm{kg}^{-1}$ & 7,81 & 54,35 \\
\hline $\mathrm{COT}_{\text {(v.s.) }}$ & dag $\mathrm{kg}^{-1}$ & 7,57 & 54,75 \\
\hline $\mathrm{N}_{\mathrm{T}}$ & $\mathrm{g} \mathrm{kg}^{-1}$ & 1,19 & 8,10 \\
\hline $\mathrm{NO}_{3}^{-}$ & $\mathrm{mg} \mathrm{kg}^{-1}$ & 62,18 & 432,71 \\
\hline $\mathrm{NH}_{4}^{+}$ & $\mathrm{mg} \mathrm{kg}^{-1}$ & 3,93 & 27,35 \\
\hline NO & $\mathrm{mg} \mathrm{kg}^{-1}$ & $1.123,89$ & $7.633,10$ \\
\hline $\mathrm{C} / \mathrm{N}$ & -0 & 50,3 & 51,8 \\
\hline $\mathrm{K}$ & $\mathrm{g} \mathrm{kg}^{-1}$ & 2,6 & 18,09 \\
\hline $\mathrm{Na}$ & $\mathrm{g} \mathrm{kg}^{-1}$ & 0,02 & 0,14 \\
\hline $\mathrm{Ca}$ & $\mathrm{g} \mathrm{kg}^{-1}$ & 0,25 & 1,74 \\
\hline $\mathrm{Mg}$ & $\mathrm{g} \mathrm{kg}^{-1}$ & 0,52 & 3,62 \\
\hline $\mathrm{P}$ & $\mathrm{g} \mathrm{kg}^{-1}$ & 0,22 & 1,53 \\
\hline ST & dag kg ${ }^{-1}$ & 14,37 & 100,0 \\
\hline SFT & dag kg $\mathrm{kg}^{-1}$ & 5,17 & 5,17 \\
\hline SVT & dag kg $\mathrm{kg}^{-1}$ & 94,83 & 94,83 \\
\hline Conteúdo de água & dag kg-1 & 85,63 & - \\
\hline
\end{tabular}

Para a caracterização física e química do CXbd, as análises foram feitas de acordo com os métodos descritos por MATOS (2012). O solo utilizado no experimento apresentou as seguintes características: $\mathrm{pH}$ em água de 5,52; 52,6 $\mathrm{mg} \mathrm{dm}^{-3} \mathrm{de} \mathrm{P}$-disp; $0,15 \mathrm{cmol}_{\mathrm{c}} \mathrm{dm}^{-3} \mathrm{de} \mathrm{K}, 0 \mathrm{cmol}_{\mathrm{c}} \mathrm{dm}^{-3} \mathrm{de}$ $\mathrm{Na}, 2,86 \mathrm{cmol}_{\mathrm{c}} \mathrm{dm}^{-3}$ de $\mathrm{Ca}+\mathrm{Mg}$ e $0,07 \mathrm{cmol}_{\mathrm{c}} \mathrm{dm}^{-3} \mathrm{de}^{3+}$ trocáveis; 7,05 $\mathrm{cmol}_{\mathrm{c}} \mathrm{dm}^{-3} \mathrm{de} \mathrm{H}+\mathrm{Al}$; soma de bases trocáveis $(\mathrm{SB})$ de $3,0 \mathrm{cmol}_{\mathrm{c}} \mathrm{dm}^{-3}$; capacidade de troca catiônica (CTC potencial a $\mathrm{pH} 7,00$ ) de $10,05 \mathrm{cmol}_{\mathrm{c}} \mathrm{dm}^{-3}$; saturação por bases (V) de $29,9 \%$; saturação por alumínio (m) de 2,28\%; $\mathrm{N}_{\mathrm{T}}$ de $1,0 \mathrm{~g} \mathrm{~kg}^{-1}$ e $\mathrm{CO}_{\text {fo }}$ de $0,81 \mathrm{dag} \mathrm{kg}^{-1}$.

A dose de resíduo aplicada ao solo, nos experimentos de campo e de laboratório, foi baseada na concentração de nitrogênio total presente no resíduo misto e em sua quantidade requerida para o cultivo intensivo da pupunheira, estabelecendo-se uma aplicação de $300 \mathrm{~kg} \mathrm{ha}^{-1}$ de $\mathrm{N}_{\mathrm{T}}$, conforme recomendado pela EMBRAPA (NEVES et al., 2007), obtendo-se, então, uma dose de aplicação igual $250 \mathrm{t} \mathrm{ha}^{-1}$ ano $^{1}$ de resíduo fresco.

O delineamento experimental adotado foi o em blocos casualizado, com parcelas subdivididas no tempo, com quatro repetições e três tratamentos (resíduo incorporado, resíduo aplicado superficialmente e solo sem adição de resíduo, também denominado controle).

O experimento de campo foi conduzido na área experimental, durante os meses de outubro de 2012 a fevereiro de 2013, totalizando quatro meses de acompanhamento do processo, sendo feitos buracos no solo para o enterrio de vasos plásticos com $20 \mathrm{~cm}$ de altura, $30 \mathrm{~cm}$ de diâmetro superior e $20 \mathrm{~cm}$ de diâmetro inferior, perfurados no fundo e nas laterais, para permitir o livre movimento de solutos entre os dois meios, os quais foram preenchidos com, aproximadamente, $10.000 \mathrm{~cm}^{3} \mathrm{do}$ 
próprio solo removido e introduzidos de forma que seu topo ficasse rente à superfície do solo. A aplicação do resíduo nos vasos foi na forma incorporada (mistura a todo o conteúdo de solo) e disposto superficialmente sobre o solo contido nos vasos. Amostras com, aproximadamente, 100 $\mathrm{cm}^{3}$ de material foram coletadas semanalmente durante o primeiro mês e, quinzenalmente, nos meses subsequentes, para a análise da concentração do carbono orgânico facilmente oxidado $\left(\mathrm{CO}_{\mathrm{fo}}\right)$ e das concentrações de nitrogênio total, nitrogênio inorgânico nas formas amoniacal $\left(\mathrm{NH}_{4}{ }^{+}\right)$e nítrica $\left(\mathrm{NO}_{3}{ }^{-}\right)$, seguindo-se o método descrito por MATOS (2012). Dados climáticos mensais de precipitação e temperatura, para o período de outubro de 2012 a março de 2013, obtidos do Instituto Nacional de Meteorologia (INMET), correspondentes à Estação Viçosa-MG (OMM: 83642), foram utilizados como informações auxiliares para a discussão dos resultados.

Em laboratório, foi feito o monitoramento da degradação do resíduo incorporado e disposto superficialmente no solo, também durante os meses de outubro a fevereiro. Para estudar o potencial de mineralização do NO, foi utilizada a técnica de STANFORD \& SMITH (1972), com modificações propostas por ALVES (1989), sendo utilizados $100 \mathrm{~g}$ de solo, misturados a igual quantidade de areia lavada e peneirada, sendo a mistura acondicionada em colunas de percolação, construídas com tubos de PVC (4 cm de diâmetro por $30 \mathrm{~cm}$ de altura). A extração do $\mathrm{N}$ mineralizado foi realizada, fazendo percolar $100 \mathrm{~mL}$ de $\mathrm{CaCl}_{2} 0,02 \mathrm{~mol} \mathrm{~L}^{-1}$ (solução extratora), aplicados lentamente em duas frações de $50 \mathrm{~mL}$. O volume de percolado de cada tubo foi medido para permitir o cálculo das quantidades de $\mathrm{N}$ mineralizado e, nessa solução efluente, foram quantificadas as concentrações de $\mathrm{N}_{-} \mathrm{NH}_{4}{ }^{+}$e de $\mathrm{N}_{-} \mathrm{NO}_{3}{ }^{-}$, por colorimetria, segundo métodos apresentados por MATOS (2012). Após a extração inicial, novas extrações foram feitas semanalmente durante um mês e depois a cada duas semanas sucessivas, totalizando 16 semanas de incubação. Cada extração foi feita seguindo-se as mesmas operações descritas anteriormente.

A mineralização de $\mathrm{CO}$ nos materiais foi medida semanalmente, sendo calculada após análise do $\mathrm{CO}_{2}$ emanado durante o período de 16 semanas de incubação. A medição do $\mathrm{CO}_{2}$ emanado foi feita utilizando-se dos respirômetros de Bartha, com adaptações no método indicado na NBR 14.283 (ABNT, 1999), ou seja, duplicação da quantidade de solo utilizado, além de se considerar que, nas parcelas experimentais em que o resíduo foi aplicado superficialmente, a quantidade de $\mathrm{CO}_{2}$ emanado constitui apenas 50\% daquela disponibilizada com a mineralização do carbono, justificando, assim, a multiplicação das quantidades medidas por dois. Para as medições, foram utilizados 12 respirômetros, 4 contendo $100 \mathrm{~g}$ de solo e $12,5 \mathrm{~g}$ de resíduo incorporado; 4 contendo $100 \mathrm{~g}$ de solo e 12,5 g de resíduo aplicado superficialmente e 4 contendo apenas solo, os quais serviram como controle. A quantificação do $\mathrm{CO}_{2}$ foi realizada com a adição de $10 \mathrm{~mL}$ de $\mathrm{KOH} 0,5$ mol L $\mathrm{L}^{-1}$ ao conduto lateral do respirômetro para absorção, já que ocorre reação do $\mathrm{CO}_{2}$ liberado com a solução adicionada. A solução de hidróxido foi renovada semanalmente, e a titulação, feita com $\mathrm{HCl} 0,5 \mathrm{~mol} \mathrm{~L}^{-1}$.

A cinética de mineralização, os potenciais de mineralização $\left(\mathrm{N}_{\text {ino(0) }}\right.$ e $\left.\mathrm{CO}_{(0)}\right)$ e os coeficientes de mineralização $(\mathrm{k})$ do $\mathrm{NO}$ e $\mathrm{CO}$ foram obtidos a partir dos valores disponibilizados acumulados de nitrogênio inorgânico e dos valores mineralizados de carbono orgânico facilmente oxidável (campo) e de $\mathrm{C}-\mathrm{CO}_{2}$ (laboratório), durante as 16 semanas de incubação do resíduo, sendo esses parâmetros obtidos por meio do ajuste dos dados às equações de regressão derivadas do modelo exponencial simples da cinética química de primeira ordem, $\mathrm{CO}_{(\min )}=\mathrm{CO}_{(0)} *\left(1-\mathrm{e}^{-\mathrm{kc}}{ }^{*} \mathrm{t}\right)$ (1) e $\mathrm{N}_{\text {ino(disp) }}=\mathrm{N}_{\text {ino(0) }} *\left(1-\mathrm{e}^{-\mathrm{kn}{ }^{*} \mathrm{t}}\right)(2)$, respectivamente, utilizadas para descrever a mineralização do carbono e do nitrogênio orgânicos e a disponibilização do nitrogênio inorgânico, tal como proposto por STANFORD \& SMITH (1972). Os valores de $\mathrm{k}_{\mathrm{C}} \mathrm{e} \mathrm{kn}$, respectivamente, coeficientes de mineralização do carbono orgânico e disponibilização do nitrogênio inorgânico, estão expressos em $\mathrm{d}^{-1}$.

Os dados de concentração de $\mathrm{CO}_{\text {fo }}, \mathrm{C}-\mathrm{CO}_{2}$ e $\mathrm{N}_{\text {ino }}$, obtidos durante o período de decomposição de 112 dias, foram utilizados diretamente, para a obtenção das frações mineralizadas observadas $\left(\mathrm{FmCO}_{(\mathrm{Ob})}\right.$ e $\left.\mathrm{FdN}_{\text {ino(ob) }}\right)$ :

$$
\mathrm{FmCO}_{(\mathrm{Ob})}=100 *\left[\left(\mathrm{CO}_{(\text {Trat)in }}-\mathrm{CO}_{(\mathrm{Cont}) \text { in }}\right)-\left(\mathrm{CO}_{(\text {Trat }) \text { fin }}-\mathrm{CO}_{(\mathrm{Cont}) \mathrm{fin}}\right)\right] /\left(\mathrm{CO}_{(\text {Trat)in }}-\mathrm{CO}_{(\mathrm{Cont}) \text { in }}\right)
$$


em que,

$\mathrm{FmCO}_{(\mathrm{ob})}(\%)$ - fração mineralizada calculada a partir das concentrações iniciais e finais observadas de carbono, tomando-se as concentrações de $\mathrm{CO}_{\text {fo }}$ (experimento de campo) e C$\mathrm{CO}_{2}$ (experimento de laboratório) iniciais do resíduo como referencial;

$\mathrm{CO}_{\text {(Trat)in }} \mathrm{CO}_{\text {(Trat)fin }}$ - Concentração de $\mathrm{CO}_{\text {fo }}$ ou C- $\mathrm{CO}_{2}\left(\right.$ dag $\left.\mathrm{kg}^{-1}\right)$ do solo/resíduo, quantificada no início e no final do experimento, respectivamente,

$\mathrm{CO}_{\text {(Cont)in }}$ e $\mathrm{CO}_{\text {(Cont)fin }}$ - Concentração de $\mathrm{CO}_{\text {fo }}$ ou $\mathrm{C}-\mathrm{CO}_{2}$ (dag $\mathrm{kg}^{-1}$ ) do solo controle, quantificada no início e no final do experimento, respectivamente.

No que se refere ao nitrogênio inorgânico, obtém-se a fração de disponibilização $\left(\mathrm{FdN}_{\mathrm{ino}(\mathrm{Ob})}\right)$ por meio de:

$\mathrm{FdN}_{\text {ino(Ob) }}=100 *\left[\left(\mathrm{~N}_{\text {ino(Trat)fin }}-\mathrm{N}_{\text {ino(Cont)fin })}\right)\left(\mathrm{N}_{\text {ino(Trat)in }}-\mathrm{N}_{\text {ino(Cont)in })}\right)\right] /(\mathrm{NO})$

em que,

$\mathrm{N}_{\text {ino(Trat)fin }}$ e $\mathrm{N}_{\text {ino(Trat)in }}$ - nitrogênio inorgânico no solo/resíduo, respectivamente, no início e no final do período de incubação da amostra $\left(\mathrm{mg} \mathrm{kg}^{-1}\right)$;

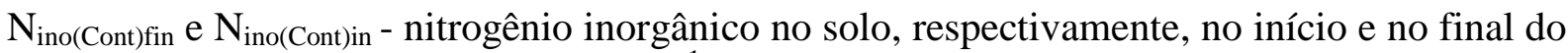
período de incubação da amostra $\left(\mathrm{mg} \mathrm{kg}^{-1}\right)$,

$\mathrm{NO}$ - nitrogênio orgânico do resíduo $\left(\mathrm{mg} \mathrm{kg}^{-1}\right)$.

Os parâmetros ajustados nas equações exponenciais de cinética de reação de primeira ordem $\left(\mathrm{CO}_{(0)}\right.$ e $\left.\mathrm{N}_{\text {ino(0) }}\right)$ e as estimativas geradas utilizando-se das eqs. (1) e (2) foram utilizadas na obtenção das frações mineralizadas. As frações mineralizadas de $\mathrm{CO}\left(\mathrm{FmCO}_{(\mathrm{Est1})}\right.$ e $\left.\mathrm{FmCO}_{(\mathrm{Est2})}\right)$ e disponibilizadas de $\mathrm{N}_{\text {ino }}\left(\mathrm{FdN}_{\text {ino(Est1) }}\right.$ e $\left.\mathrm{FdN}_{\text {inorg(Est2) }}\right)$ foram calculadas utilizando-se das seguintes equações:

$$
\begin{aligned}
& \mathrm{FmCO}_{(\text {Est1) }} 100 * \mathrm{CO}_{(\mathrm{min})} / \mathrm{CO}_{0} \\
& \mathrm{FmCO}_{(\text {Est2) }}=100 * \mathrm{CO}_{(\text {min) }} / \mathrm{CO}_{\mathrm{fo}} \\
& \mathrm{FdN}_{\text {ino(Est1) }}=100 * \mathrm{~N}_{\text {inorg(disp })} / \mathrm{N}_{\text {ino(0) }} \\
& \mathrm{FdN}_{\text {ino(Est2) }}=100 * \mathrm{~N}_{\text {inorg(disp) }} / \mathrm{NO}
\end{aligned}
$$

em que,

$\mathrm{CO}_{\text {fo }}$ e NO - respectivamente, concentração de carbono orgânico facilmente oxidável e nitrogênio orgânico do resíduo ( $\mathrm{dag} \mathrm{kg}^{-1}$ ou $\mathrm{mg} \mathrm{kg}^{-1}$ ).

\section{RESULTADOS E DISCUSSÃO}

O modelo exponencial simples de cinética de primeira ordem ajustou-se bem a todos os dados obtidos, para um nível de significância mínimo dos coeficientes de 1\%, estando as curvas ajustadas e suas respectivas equações apresentadas nas Figuras 1 e 2. 


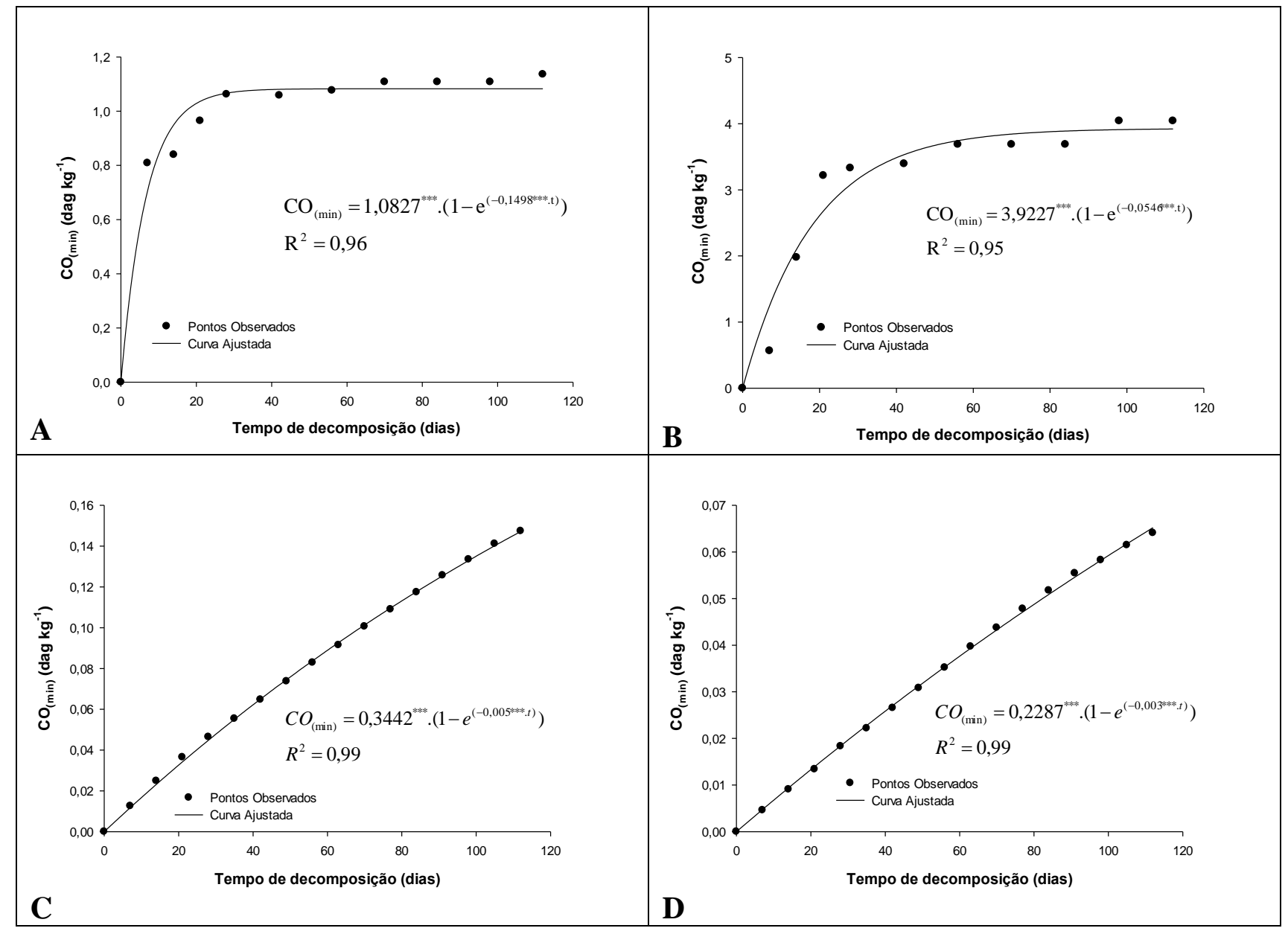

FIGURA 1. Concentração acumulada de carbono orgânico mineralizado $\left(\mathrm{CO}_{(\min )}\right)$ para a condição de campo do resíduo incorporado (A) e aplicado superficialmente no solo (B) e para a condição de laboratório do resíduo incorporado (C) e aplicado superficialmente no solo (D) e suas equações ajustadas para um período de 112 dias de monitoramento. Cumulative mineralized organic carbon $\left(\mathrm{OC}_{(\mathrm{min})}\right)$ under field conditions for incorporated (A) and surface-applied waste (B), as well as under lab conditions for incorporated (C) and surface-applied waste (D), and equations adjusted for a 112-day monitoring period. 


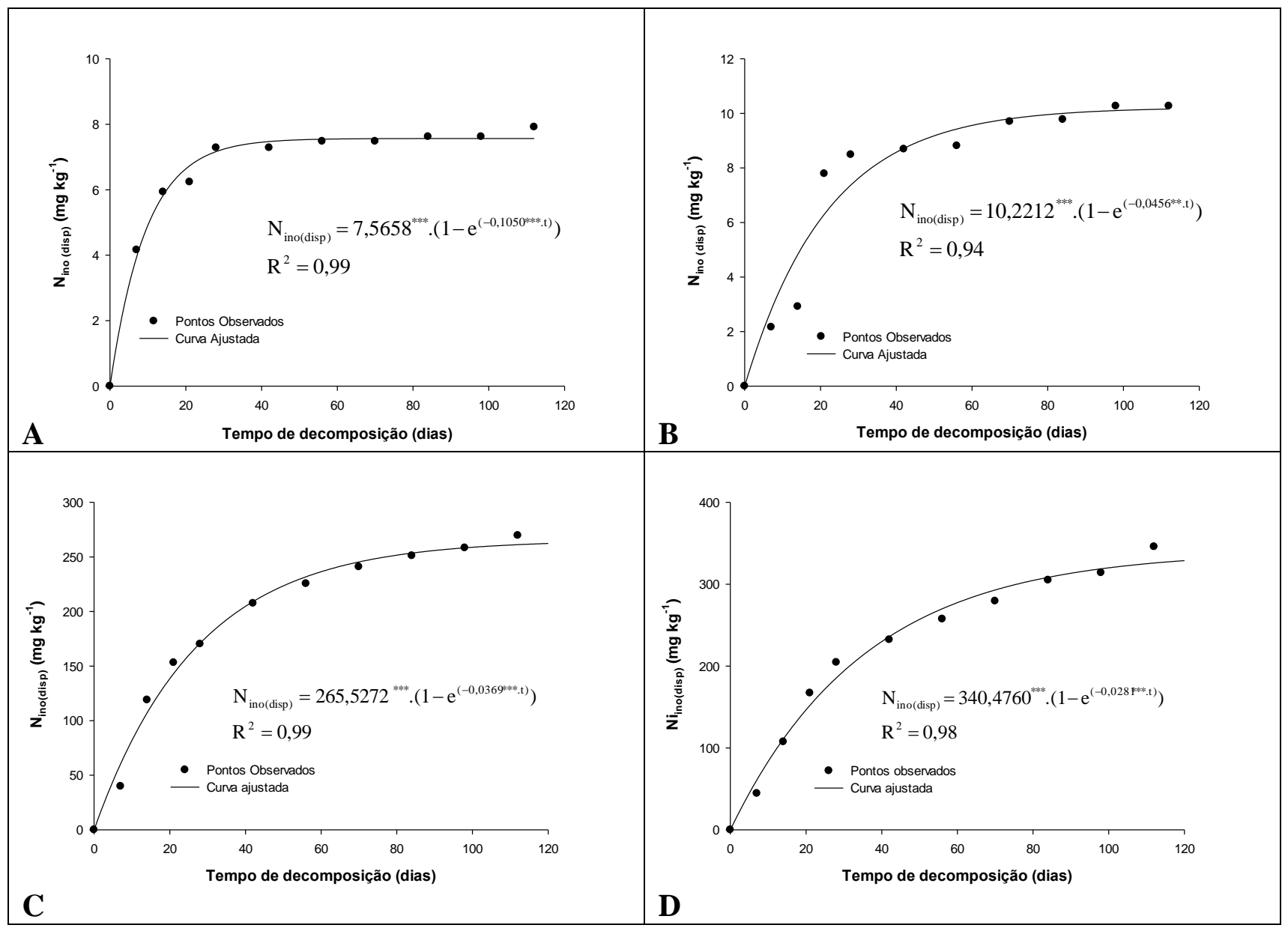

FIGURA 2. Concentração acumulada de nitrogênio inorgânico disponibilizado ( $\mathrm{N}_{\text {ino(disp) }}$ ) para a condição de campo do resíduo incorporado (A) e aplicado superficialmente no solo (B) e para a condição de laboratório do resíduo incorporado (C) e aplicado superficialmente no solo (D) e suas equações ajustadas para um período de 112 dias de monitoramento. Cumulative available inorganic nitrogen $\left(\mathbf{N}_{\text {ino(disp) }}\right)$ under field conditions for incorporated (A) and surface-applied waste (B), as well as under lab conditions for incorporated (C) and surface-applied waste (D), and equations adjusted for a 112-day monitoring period.

Nas Tabelas 2 e 3, estão apresentados os valores de carbono orgânico potencialmente mineralizável $\left(\mathrm{CO}_{(0)}\right)$, carbono orgânico mineralizado acumulado $\left(\mathrm{CO}_{(\mathrm{min})}\right)$, nitrogênio inorgânico potencialmente disponibilizável $\left(\mathrm{N}_{\mathrm{ino}(0)}\right)$, nitrogênio inorgânico disponibilizado acumulado $\left(\mathrm{N}_{\text {ino(disp) }}\right)$ e as diferentes formas de cálculo para frações de mineralização do $\mathrm{CO}$ e disponibilização

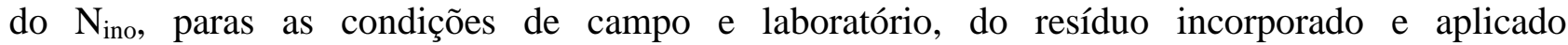
superficialmente. Nota-se que tanto na condição de campo como na de laboratório, a incorporação do resíduo ao solo acelerou consideravelmente seu processo de degradação, fazendo com que o kc e o kn do resíduo incorporado fossem aproximadamente 2,2 e 1,8 vezes maiores, respectivamente, que o do resíduo aplicado superficialmente. Outro fato é que os valores de kc e kn para a condição de campo foram, respectivamente, 24,0 e 2,2 vezes maiores do que os obtidos em condição de laboratório.

Os valores de kc e kn, obtidos em um experimento de campo, por GAMA-RODRIGUES et al. (2007) para os restos das culturas de feijão-de-porco, amendoim forrageiro, siratro, kudzu, braquiária adubada e braquiária não adubada, foram inferiores aos obtidos neste trabalho para o resíduo da pupunheira, o que pode ter ocorrido devido a diferenças na metodologia adotada, na qual amostras de $30 \mathrm{~g}$ e $10 \mathrm{~g}$ dos resíduos culturais (sem serem picados) foram colocadas em sacos de náilon (malha $2 \mathrm{~mm}$ ) e estes dispostos sobre a superfície do solo, e o ajuste feito com base nos valores remanescentes de $\mathrm{CO}$ e NO. A utilização, pelos autores, de materiais não picados e 
dispostos dentro de sacos sobre o solo, pode ter sido, no entanto, o fator que mais contribuiu para os menores valores encontrados, visto que a acessibilidade dos macro (formigas, cupins, anelídeos...), meso (pequenos insetos, ácaros, colêmbolos...) e microrganismos foi comprometida. As frações mineralizadas do $\mathrm{CO}$ e do $\mathrm{NO}$ foram inferiores, porém próximas aos valores encontrados para o resíduo misto da pupunheira, para tempos semelhantes de monitoramento do processo. Ademais, a menor fração mineralizada obtida por eles foi de $44,54 \%$ (braquiária não adubada), indicando que, mesmo quando dispostos superficialmente, no mínimo $40 \%$ dos resíduos foram mineralizados até o terceiro/quarto mês de sua disposição no campo, evidenciando a rápida degradação/mineralização de resíduos sólidos orgânicos nas condições climáticas brasileiras.

TABELA 2. Parâmetros das equações de cinética de primeira ordem da degradação do carbono orgânico $(\mathrm{CO})$, coeficiente de mineralização $(\mathrm{kc})$ e fração mineralizada (FmCO), obtidos a partir dos ajustes dos dados de carbono orgânico mineralizado acumulado, em solos nos quais os resíduos foram incorporados ou aplicados superficialmente, após 112 dias de monitoramento, para as condições de campo e laboratório. Parameters of first-order kinetics equation $^{(1)}$ for organic carbon (OC) degradation, mineralization coefficient (kc) and mineralized fraction (FmOC) derived from adjusted data of cumulative mineralized organic carbon, wherein wastes were incorporated or applied onto the soil surface, after a 112-day monitoring period under field and lab conditions.

\begin{tabular}{|c|c|c|c|c|c|c|c|c|}
\hline \multicolumn{2}{|c|}{ Forma de aplicação } & $\frac{\mathrm{CO}_{(0)}}{\left(\mathrm{dag} \mathrm{kg}^{-1}\right)}$ & $\frac{\mathrm{kc}}{\left(\mathrm{d}^{-1}\right)}$ & $\frac{\mathrm{R}^{2}}{-}$ & $\frac{\mathrm{CO}_{(\min )}}{\left(\mathrm{dag} \mathrm{kg}^{-1}\right)}$ & $\frac{\mathrm{FmCO}_{(\mathrm{ob})}{ }^{(1)}}{(\%)}$ & $\frac{\mathrm{FmCO}_{(\text {Est1 })}{ }^{(2)}}{(\%)}$ & $\frac{\mathrm{FmCO}_{(\text {Est2) }}{ }^{(3)}}{(\%)}$ \\
\hline \multirow{2}{*}{ Campo } & Incorporado & $1,08 * * *$ & $0,1498 * * *$ & 0,9689 & 1,08 & 93,47 & 100,00 & 100,00 \\
\hline & Superficial & $3,92 * * *$ & $0,0546 * * *$ & 0,9516 & 3,91 & 59,82 & 99,78 & 67,26 \\
\hline \multirow{2}{*}{ Laboratório } & Incorporado & $0,344 * * *$ & 0,0050 *** & 0,9992 & 0,147 & 50,51 & 42,88 & 2,45 \\
\hline & Superficial & $0,228 * * *$ & $0,0030 * * *$ & 0,9993 & 0,065 & 36,01 & 24,42 & 1,07 \\
\hline
\end{tabular}

$\overline{\mathrm{ns}, * * *, * * * *}$ Não significativo e significativos em nível de $0,1 \%, 1 \%$ e $5 \%$ de probabilidade, respectivamente.

$\mathrm{CO}_{(0)}$, carbono orgânico potencialmente mineralizável do resíduo, em dag $\mathrm{kg}^{-1} ; \mathrm{CO}_{(\min )}$, carbono orgânico facilmente oxidável mineralizado e acumulado durante os 112 dias de experimento, em dag kg ${ }^{-1}$; $\mathrm{kc}$, coeficiente de mineralização do carbono orgânico do resíduo, em dias ${ }^{-1} ; \mathrm{R}^{2}=$ coeficiente de determinação; Fração de mineralização: ${ }^{(1)} \mathrm{FmCO}_{(\mathrm{ob})}=100 \times\left[\left(\mathrm{CO}(\mathrm{Trat})_{\text {in }}-\mathrm{CO}(\mathrm{Cont})_{\text {in }}\right)-\right.$ $\left.\left(\mathrm{CO}(\text { Trat })_{\mathrm{fin}}-\mathrm{CO}(\mathrm{Cont})_{\mathrm{fin}}\right)\right] /\left(\mathrm{CO}(\text { Trat })_{\mathrm{in}}-\mathrm{CO}(\mathrm{Cont})_{\mathrm{in}}\right) ;{ }^{(2)} \mathrm{FmCO}_{(\mathrm{Est} 1)}=100 \mathrm{x}\left(\mathrm{CO}_{(\mathrm{min})} / \mathrm{CO}_{(0)}\right) ;{ }^{(3)} \mathrm{FmCO}_{(\mathrm{Est} 2)}=100 \times(\mathrm{CO})$

TABELA 3. Parâmetros das equações de cinética de primeira ordem da degradação do nitrogênio orgânico $(\mathrm{NO})$, coeficiente de disponibilização $(\mathrm{kn})$ e fração disponibilizada $\left(\mathrm{FdN}_{\mathrm{ino}}\right)$, obtidos a partir dos ajustes dos dados de nitrogênio inorgânico acumulado, em solos nos quais os resíduos foram incorporados ou aplicados superficialmente, após 112 dias de monitoramento para as condições de campo e laboratório. Parameters of firstorder kinetics equation ${ }^{(1)}$ for organic nitrogen $(\mathrm{ON})$ degradation, availability coefficient (kn) and available fraction (FdNino) derived from adjusted data of cumulative inorganic nitrogen, wherein wastes were incorporated or applied onto the soil surface, after a 112-day monitoring period under field and lab conditions.

\begin{tabular}{lcccccccc}
\hline \multirow{2}{*}{ Forma de aplicação } & $\mathrm{N}_{\text {ino(0) }}$ & $\mathrm{kn}$ & $\mathrm{R}^{2}$ & $\mathrm{~N}_{\text {ino(disp) }}$ & $\mathrm{FdN}_{\text {ino(ob) }}{ }^{(1)}$ & $\mathrm{FdN}_{\text {ino(Est1) }}{ }^{(2)}$ & $\mathrm{FdN}_{\text {ino(Est2) }}{ }^{(3)}$ \\
\cline { 2 - 9 } & $\left(\mathrm{mg} \mathrm{kg}^{-1}\right)$ & $\left(\mathrm{d}^{-1}\right)$ & - & $\left(\mathrm{mg} \mathrm{kg}^{-1}\right)$ & $(\%)$ & $(\%)$ & $(\%)$ \\
\hline \multirow{2}{*}{ Campo } & Incorporado & $7,56^{* * *}$ & $0,1050^{* * *}$ & 0,9910 & 7,56 & 95,27 & 100,00 & 7,75 \\
& Superficial & $10,22^{* *}$ & $0,0456^{* *}$ & 0,9405 & 10,16 & 62,66 & 99,39 & 0,91 \\
\hline \multirow{2}{*}{ Laboratório } & Incorporado & $265,53 * * *$ & $0,0369 * * *$ & 0,99 & 261,27 & 84,37 & 98,40 & 24,00 \\
& Superficial & $340,48^{* * *}$ & $0,0281 * * *$ & 0,98 & 325,84 & 62,09 & 95,70 & 30,76 \\
\hline
\end{tabular}

ns, ${ }^{* * *,},{ }^{* *},{ }^{*}$ Não significativo e significativo em nível de $0,1 \%, 1 \%$ e $5 \%$ de probabilidade, respectivamente.

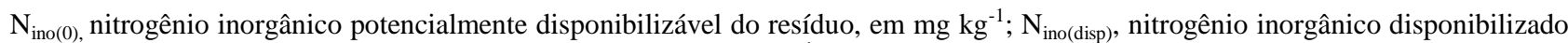
acumulado durante os 112 dias de incubação do resíduo, em mg kg${ }^{-1} ; \mathrm{kn}$, coeficiente de disponibilização do nitrogênio inorgânico do resíduo, em dias ${ }^{-1} ; \mathrm{R}^{2}=$ coeficiente de determinação; Fração de mineralização: ${ }^{(1)} \mathrm{FdN}_{\text {ino(ob) }}=100 \mathrm{x}\left[\left(\mathrm{N}_{\text {ino(Trat }) \text { in }}{ }^{-} \mathrm{N}_{\text {ino(Cont)in }}\right)\right.$ $\left.\left(\mathrm{N}_{\text {ino(Trat)fin }}-\mathrm{Nino}_{(\mathrm{Cont}) \mathrm{fin}}\right)\right] /\left(\mathrm{N}_{\text {ino(Trat)in }}-\mathrm{N}_{\text {ino(Cont)in }}\right) ;{ }^{(2)} \mathrm{FmCO}_{(\text {Est } 1)}=100 \times\left(\mathrm{CO}_{(\min )} / \mathrm{CO}_{(0)}\right) ;{ }^{(3)} \mathrm{FdN}_{\text {ino(Est2) }}=100 \times\left(\mathrm{N}_{\text {ino(disp })} / \mathrm{NO}\right)$.

As frações mineralizadas do $\mathrm{CO}$, em condição de campo, para o resíduo incorporado, calculadas com base nas equações 5 e 6 , foram bem próximas e superiores à que foi obtida 
utilizando-se da Equação 3. No que se refere ao resíduo aplicado superficialmente, as frações calculadas com base nas equações 3 e 6 foram próximas e inferiores à obtida utilizando-se da Equação 5. Isto indica que a utilização da Equação 3 tende a superestimar o valor das frações de mineralização. As diferenças encontradas na estimativa das frações mineralizadas de CO, utilizando-se dos três métodos, podem ser consideradas pequenas, o que viabiliza a utilização de todos os três para a obtenção desses indicadores de mineralização do material orgânico dos resíduos da pupunheira. Contudo, considera-se que os resultados obtidos, utilizando-se da concentração de carbono orgânico presente no resíduo, sejam mais confiáveis e verossímeis.

No que se refere às frações obtidas considerando-se o $\mathrm{N}_{\text {ino, }}$ as equações 4 e 7 forneceram resultados próximos, entretanto bem superiores aos obtidos pela Equação 8, indicando possivelmente que pequena parte do $\mathrm{N}_{\text {ino }}$ disponibilizado tenha sido recuperado nas análises das amostras, o que repercutiu em baixas frações mineralizadas, estando esse fato possivelmente associado à maior instabilidade do $\mathrm{N}$ no meio, principalmente devido a sua alta propensão de ser lixiviado no perfil do solo sob altos regimes pluviométricos.

Com base nos dados das Tabelas 2 e 3, pode-se concluir que mais de $90 \%$ do resíduo incorporado e mais de $50 \%$ do resíduo disposto na superfície, em condição de campo, foram mineralizados nos 112 dias de sua incubação. Isso indica que a maior parte do resíduo foi decomposta dentro dos trinta primeiros dias, e que a incorporação proporcionou aumento expressivo na fração mineralizada do resíduo. A rápida mineralização do resíduo pode ser explicada pela grande labilidade do material orgânico em estudo e em virtude da pequena granulometria do material (após passado em picadeira, tinha dimensões de 3 a $6 \mathrm{~mm}$ ), o que aumentou consideravelmente sua superfície específica e suscetibilidade à ação dos meso e micro-organismos e aos altos índices pluviométricos observados durante o tempo do experimento (Tabela 4).

TABELA 4. Dados climáticos observados durante o período de condução do experimento. Climatic data recorded during experiment conduct.

\begin{tabular}{lcccc}
\hline \multirow{2}{*}{ Meses } & \multirow{2}{*}{ Precipitação $(\mathrm{mm})$} & \multicolumn{3}{c}{ Temperatura $\left({ }^{\circ} \mathrm{C}\right)$} \\
\cline { 3 - 5 } & & Máxima & Média & Mínima \\
\hline Outubro & 88,9 & 29,6 & 21,8 & 16,0 \\
Novembro & 235,4 & 26,4 & 21,3 & 18,3 \\
Dezembro & 199,3 & 30,8 & 23,9 & 19,5 \\
Janeiro & 143,1 & 28,2 & 22,5 & 19,0 \\
Fevereiro & 109,6 & 29,4 & 22,5 & 18,2 \\
\hline
\end{tabular}

Fonte: INMET (2013).

DONEDA et al. (2012) obtiveram valores do coeficiente de mineralização da fração lábil da matéria seca $(\mathrm{k})$, variando de 0,0525 a $0,0752 \mathrm{~d}^{-1}$, dos restos culturais de várias plantas de cobertura de outono-inverno (centeio, aveia-preta, ervilhaca forrageira, nabo forrageiro e ervilhaca comum) dispostas sobre o solo em um experimento de campo com duração de 164 dias, na região de Mantiqueira-RS. Os valores encontrados por eles foram bem próximos aos encontrados neste trabalho para os resíduos dispostos sobre o solo, e segundo o exposto pelos autores, em torno de $50 \%$ de todos os restos culturais mineralizaram com menos de 30 dias, fato também semelhante ao obtido para os resíduos da pupunheira.

Em um trabalho feito em casa de vegetação, na cidade de Petrolina-PE, GIONGO et al. (2011) obtiveram valores de kc variando de 0,0103 a $0,0123 \mathrm{~d}^{-1}$ e de $\mathrm{kn}$ variando de 0,0088 a 0,012 $\mathrm{d}^{-1}$, de coquetéis preparados com os restos culturais de várias plantas leguminosas e não leguminosas, utilizadas como adubo verde ou de cobertura, em diferentes proporções, dispostos sobre o solo e com um tempo de experimento de 210 dias. Os menores valores de kc e kn possivelmente se devem novamente às diferenças nas metodologias, nos modelos matemáticos adotados e, principalmente, nas características constitucionais das plantas avaliadas. Contudo, fica evidente a rápida mineralização dos resíduos vegetais quando incorporados ou dispostos sobre o 
solo, visto que $50 \%$ de todos os coquetéis preparados pelos autores mineralizaram em torno de 60 dias após o início do experimento.

$\mathrm{Na}$ condição de campo, a incorporação dos resíduos ao solo acelerou consideravelmente o processo de degradação do material orgânico, fazendo com que a fração mineralizada se tornasse, aproximadamente, duas vezes maior que a dos resíduos dispostos sobre a superfície do solo. $\mathrm{O}$ resíduo incorporado mineralizou mais rapidamente que o aplicado superficialmente, possivelmente devido ao aumento do contato das partículas do resíduo com o solo, ocasionado pela sua mistura e homogeneização com o mesmo; manutenção de maiores valores de conteúdo de água no solo por mais tempo dentro dos vasos, comparativamente aos encontrados na superfície do solo: e a não incidência de radiação solar direta sobre o resíduo incorporado, que sabidamente é nociva aos micro-organismos que participam no processo de degradação do resíduo. Este fato também foi observado no experimento em laboratório, possivelmente devido ao maior contato do resíduo com o solo e à conservação da umidade da mistura solo-resíduo.

Em um experimento de laboratório, BARRETO et al. (2010) estimaram os valores de $\mathrm{CO}_{(0)}$, $\mathrm{kc}, \mathrm{FmCO}_{(\mathrm{Est} 1)}, \mathrm{N}_{\text {ino(0) }}$, $\mathrm{kn}$ e $\mathrm{FmNO}_{(\mathrm{Est1})}$, de amostras de serapilheira de plantações de eucalipto de 1; 3; 5 e 13 anos, quando incorporadas ao solo. Comparativamente aos valores obtidos neste trabalho para o resíduo da pupunheira, os valores de kc obtidos por eles foram superiores, mas os valores de $\mathrm{CO}_{(0)}, \mathrm{N}_{\text {ino(0) }}$ e kn foram inferiores, o que pode ter sido acarretado pelas diferenças existentes na constituição dos resíduos (relação $\mathrm{C} / \mathrm{N}$, concentração de celulose, lignina, etc.), pelo maior tempo de monitoramento do processo, pela possível exaustão do oxigênio dentro das câmaras ou pelas diferenças entre os métodos empregados. As frações mineralizadas obtidas por eles ficaram próximas às obtidas neste trabalho, para o resíduo incorporado, com valores médios de $\mathrm{FmCO}_{(\mathrm{Est1})} \mathrm{e}$ $\mathrm{FmNO}_{(\mathrm{Est1})}$ de $92,4 \%$ e $76,5 \%$, respectivamente. O mesmo autor também relatou que a maior parte do carbono e do nitrogênio orgânicos da serapilheira foram mineralizados durante as primeiras semanas de incubação, o que repercutiu em maiores coeficientes no início e em menores coeficientes no final do processo, devido à permanência de compostos de mais difícil degradação ao longo do processo.

LI et al. (2012) estimaram os coeficientes de mineralização dos restos culturais de soja e milho, incorporados e dispostos sobre o solo, num experimento de laboratório, com tempo de incubação de 56 dias, com valores de kc de 0,053 e 0,027 $\mathrm{d}^{-1}$ para aos restos culturais de soja e milho incorporados no solo, respectivamente. Quando dispostos sobre o solo, os valores de kc foram de 0,073 e 0,047 $\mathrm{d}^{-1}$ para aos restos culturais de soja e milho, respectivamente. Contrariamente ao obtido neste trabalho, os resíduos dispostos sobre a superfície mineralizaram mais rapidamente que os incorporados ao solo, o que, segundo os autores, pode ter ocorrido devido à menor disponibilidade de $\mathrm{O}_{2}$ para os microrganismos aeróbios dentro do solo. A coleta e o preparo de amostras de solo para incubação em laboratório, como a quebra dos agregados e o peneiramento do solo, podem contribuir para a menor macroporosidade do solo incubado, repercutindo assim em menores trocas gasosas entre o interior do solo e o ambiente.

CARNEIRO et al. (2013) incubaram em laboratório, durante 270 dias, amostras de diversos resíduos orgânicos em dois tipos de solo, $\mathrm{LVd}$ e $\mathrm{LVA}$, obtendo valores de $\mathrm{kn}$ variando de $0,0075 \mathrm{~d}^{-1}$ a $0,0143 \mathrm{~d}^{-1}$ no $L V d$ e valores de kn variando de $0,001 \mathrm{~d}^{-1}$ a $0,01 \mathrm{~d}^{-1}$ no LVA. Pelos valores obtidos pelos autores, fica evidente a grande variação existente nos valores estimados de $\mathrm{kn}$, principalmente entre os dois tipos de solos utilizados, fato devido aos inúmeros fatores $(\mathrm{pH}$, temperatura, conteúdo de água, microbiota, características dos resíduos e dos solos) que interferem no processo de mineralização. Contudo, análogo ao obtido neste trabalho, os autores relataram que houve rápida mineralização do NO de vários resíduos nas primeiras semanas de incubação.

Os valores de $\mathrm{CO}_{(0)}$ e $\mathrm{FmCO}_{(\mathrm{Est1})}$ encontrados por DOSSA et al. (2008), das partes aéreas de duas espécies arbóreas, Nioro (P. reticulatum) e Keur Mata (G. senegalensis), incorporadas ao solo e incubadas em laboratório, por um período de 118 dias, foram semelhantes aos valores encontrados neste trabalho para o resíduo misto da pupunheira, enquanto os valores de kc encontrados por eles foram bem superiores. Possivelmente, isso tenha ocorrido devido às diferenças existentes na 
constituição dos resíduos avaliados, na metodologia empregada e nas condições de incubação utilizadas, principalmente no controle da temperatura, que têm influência direta na taxa/coeficientes de mineralização de materiais orgânicos. QUADRO et al. (2011), em um experimento de laboratório e um período de incubação de 160 dias, obtiveram frações de mineralização que variaram de 75 a $100 \%$, para diferentes doses de dejetos de suínos $\left(0 ; 6 ; 12 ; 18 ; 24\right.$ e $\left.30 \mathrm{t} \mathrm{ha}^{-1}\right)$, incorporadas ao solo com e sem calagem, corroborando o que foi obtido neste trabalho.

Examinando-se os valores apresentados nas Tabelas 2 e 3, pode ser observado que os coeficientes de mineralização estimados para o experimento de campo foram muito superiores aos estimados para o experimento de laboratório. Os valores estimados de kc para o resíduo incorporado e disposto superficialmente, em condição de campo, foram em torno de, respectivamente, 30 e 20 vezes maiores que os valores de $\mathrm{kc}$ do resíduo incorporado e disposto superficialmente, em condição de laboratório. Já em relação aos valores de kn, estimou-se que, no resíduo incorporado e disposto superficialmente, em condição de campo, eles foram cerca de 2,8 e 1,6 vezes maiores, respectivamente, que o incorporado e disposto superficialmente, em condição de laboratório.

Esses resultados devem-se à existência, nas condições de campo, de fatores como maior volume de solo para contato com o resíduo, o que proporcionou maior representatividade das condições reais de disposição de resíduos orgânicos nesse meio; ser, o sistema, aberto, o que possibilita livre fluxo de solutos entre os meios; possibilidade de maior interação do resíduo com macro e meso-organismos do solo, também atuantes ativos no processo de decomposição; e estresse e tempo de adaptação dos microrganismos às condições de laboratório (temperatura, umidade, pressão e concentração de gases), o que certamente propicia taxas de mineralização diferentes das obtidas em condição de campo.

Um problema ocorrente em experimentos conduzidos em laboratório, com incubação das amostras no interior de aparatos em que há contenção dos gases, é a dificuldade de manutenção de uma concentração mínima de oxigênio no meio, durante todo o tempo entre uma amostragem e outra, sendo que, em muitos casos, pode ocorrer sua completa exaustão, concorrendo para que o processo de degradação do material orgânico deixe de ser aeróbio e passe a ser anaeróbio/anóxico, sabidamente mais lento. A manutenção da temperatura ambiente em $30^{\circ} \mathrm{C}$ (DOSSA et al., 2008), durante todo o tempo de incubação do resíduo, constitui condição irreal e não representativa do que ocorre em campo, podendo resultar na obtenção de valores de kc e frações mineralizadas diferentes.

Atualmente, a maioria dos experimentos sobre a mineralização do $\mathrm{CO}$ e $\mathrm{NO}$ de resíduos orgânicos é realizada ex-situ (em laboratório) por serem de mais fácil instalação e operação; contudo, muitas vezes, não conseguem abarcar uma infinidade de fatores que podem influenciar o processo in-situ (em campo), como, por exemplo, a variação diurna de temperatura e teor de água do solo, que podem desempenhar um papel vital na mineralização dos resíduos (GLANVILLE et al., 2012). Com base nisso, considera-se que os experimentos de laboratório seriam mais indicados apenas para uma pré-avaliação dos processos, subsidiando, assim, estudos posteriores em escala de campo, que ainda se fazem necessários na maioria dos casos de disposição e/ou aproveitamento de resíduos orgânicos no solo.

\section{CONCLUSÕES}

A incorporação dos resíduos gerou frações mineralizadas superiores às obtidas com disposição superficial, tendo sido obtidos valores médios de $96,5 \%$ e 79,0\%, respectivamente, aos 112 dias de monitoramento.

A condição de campo foi a que gerou melhores resultados no estudo de degradação dos resíduos da pupunheira, sendo que a avaliação da mineralização do $\mathrm{CO}$, por ser mais estável e menos suscetível às variações climáticas, foi a mais apropriada para a estimativa dos coeficientes e frações de mineralização do referido resíduo. 


\section{REFERÊNCIAS}

ABNT - ASSOCIAÇÃO BRASILEIRA DE NORMAS TÉCNICAS. NBR 14.283: Rio de Janeiro, 1999. 8p.

BARRETO, P.A.B.; GAMA-RODRIGUES, E.F.; GAMA-RODRIGUES, A.C.; BARROS, N.F.; ALVES, B.J. R.; FONSECA, S. Mineralização de nitrogênio e carbono em solos sob plantações de eucalipto, em uma sequência de idades. Revista Brasileira de Ciência do Solo, Viçosa, MG, v.34, n.3, p.735-745, 2010.

BARROS, F.M.; MARTINEZ, M.A.; MATOS, A.T.; NEVES, J.C.L.; SILVA, D.D.; Parametrização de modelos de mineralização do nitrogênio orgânico com água residuária de suinocultura. Revista Ambiente \& Água, Taubaté, v.5, n. 2, p.99-111, 2010.

BOEIRA, R.C.; LIGO, M.A.V.; MAXIMILIANO, V.C.B; PIRES, A.M.M. Determinação da fração de mineralização de compostos nitrogenados de lodos de esgoto aplicados em solo agrícola. Jaguariúna: EMBRAPA Meio Ambiente, 2011. 5p. (Circular Técnica, 20).

CARNEIRO, W.J.O.; SILVA, C.A.; MUNIZ, J.A.; SAVIAN, T.V. Mineralização de nitrogênio em latossolos adubados com resíduos orgânicos. Revista Brasileira de Ciência do Solo, Viçosa, MG, v.37, n.3, p.715-725, 2013.

DONEDA, A; AITA, C.; GIACOMINI, S.J.; MIOLA, E.C.C.; GIACOMINI, D.A.; SCHIRMANN, J.; GONZATTO, R. Fitomassa e decomposição de resíduos de plantas de cobertura puras e consorciadas. Revista Brasileira de Ciência do Solo, Viçosa, MGv.36, n.6, p.1714-1723, 2012.

DOSSA, E.L.; KHOUMA, M.; DIEDHIOU, I.; SENE, M.; KIZITO, F.; BADIANE, A.N.;

SAMBA, S.A.N.; DICK, R.P. Carbon, nitrogen and phosphorus mineralization potential of semiarid Sahelian soils amended with native shrub residues. Geoderma, Amsterdam, v.148. p.251-260, 2008.

FIGUEIREDO, C.C.; RAMOS, M.L.G.; MCMANUS, C.M.; MENEZES, A.M. Mineralização de esterco de ovinos e sua influência na produção de alface. Horticultura Brasileira, Vitória da Conquista, v.30, n.1, p.175-179, 2012.

FIORESE, C.; CERETTA, C.A.; GIACOMINI, S. J.; LORENSINI, F.; TRENTIN, G. Liberação do $\mathrm{N}$ em solos de diferentes texturas com ou sem adubos orgânicos. Ciência Rural, Santa Maria, v.42, n.7, p.1187-1192, jul, 2012.

GAMA-RODRIGUES, A.C.; GAMA-RODRIGUES, E.F.; BRITO, E.C. Decomposição e liberação de nutrientes de resíduos culturais de plantas de cobertura em argissolo vermelho-amarelo na região noroeste fluminense (RJ). Revista Brasileira de Ciência do Solo, Viçosa, MG, v.31, n.6, p.14211428, 2007.

GIONGO, V.; MENDES, A.M.S.; CUNHA, T.J.F.; GALVÃO, S.R.S. Decomposição e liberação de nutrientes de coquetéis vegetais para utilização no Semiárido brasileiro. Revista Ciência Agronômica, Fortaleza, v.42, n.3, p. 611-618, jul./set., 2011.

GLANVILLE, H.; ROUSK, J.; GOLYSHIN, P.; JONES, D.L. Mineralization of low molecular weight carbon substrates in soil solution under laboratory and field conditions. Soil Biology \& Biochemistry, Amsterdam, v.48, p.88-95, 2012.

INMET - INSTITUTO NACIONAL DE METEOROLOGIA. Dados meteorológicos para ensino e pesquisa. Eixo Monumental Sul Via S1 - Sudoeste. Brasília, 2013.

TASSO Jr., L.C.T.; MARQUES, M.O.; FRANCO, A.; NOGUEIRA, G.A.; NOBILE, F.O.; CAMILOTTI, F.; SILVA, A. R. Produtividade e qualidade de cana-de-açúcar cultivada em solo tratado com lodo de esgoto, vinhaça e adubos minerais. Engenharia Agrícola, Jaboticabal, v.27, n.1, p.276-283, jan./abr. 2007. 
LI, L.J.; HAN, X.Z.; YOU, M.Y.; YUAN, Y.R.; DING, X.L.; QIAO, Y.F. Carbon and nitrogen mineralization patterns of two contrasting crop residues in a Mollisol: Effects of residue type and placement in soils. European Journal of Soil Biology, Paris, v.54, p.1-6, 2013.

MARIANO, E.; TRIVELIN, P.C.O.; LEITE, J.M.; MEGDA, M.X.V.; OTTO, R.; FRANCO, H.C. J. Incubation methods for assessing mineralizable nitrogen in soils under sugarcane. Revista Brasileira de Ciência do Solo, Viçosa, MG, v.37, n.2, p.450-461, 2013.

MATOS, A.T. Poluição ambiental - Impactos no meio físico. Viçosa: Imprensa Universitária, UFV, 2010. 260p.

MATOS, A.T. Qualidade do meio físico - Práticas de laboratório. Viçosa: Imprensa Universitária, UFV, 2012. 150p.

MATOS, A.T. Tratamento e aproveitamento agrícola de resíduos sólidos. Viçosa: Imprensa Universitária, UFV, 2014. 176p.

MORAES, J.E.; PAULINO, V.T.; POSSENTI, R. Nutrientes e perdas de matéria seca da silagem do resíduo de agroindustrial de palmito pupunha com polpa cítrica. Pesquisa \& Tecnologia, Campinas, v.8, n.2, jul./dez. 2011.

NEVES, E.J.M.; SANTOS, A.F.; RODIGHERI, H.R.; CORRÊA Jr., C.; BELLETTINI, S; TESSMANN, D.J. Cultivo da pupunheira para palmito nas regiões Sudeste e Sul do Brasil. Colombo: EMBRAPA Florestas, 2007. 9p. (Circular Técnica, 143).

QUADRO, M.S.; CASTILHOS, D.D.; CASTILHOS, R.M.V.; VIVIAN, G. Biomassa e atividade microbiana em solo acrescido de dejeto suíno. Revista Brasileira de Agrociência, Pelotas, v.17, n.1-4, p.85-93, jan./mar. 2011.

SILVA, A.A.; COSTA, A.M.; LANA, R.M.Q.; LANA, A.M. Q. Recycling of nutrients with application of organic waste in degraded pasture. Engenharia Agrícola, Jaboticabal, v.32, n.2, p.405-414, mar./abr. 2012.

SOUSA, E.P.; SOARES, N.S.; CORDEIRO, S.A.; SILVA, M.L. Competitividade da Produção de Palmito de Pupunha no Espírito Santo e em São Paulo. RESR, Piracicaba, v.49, n.1, p.157-180, jan./mar. 2011.

STANFORD G.; SMITH, S.J. Nitrogen mineralization potential of soils. Soil Science Society of American Journal, Madison, v.36, n.3, p.465-472, 1972. 